\title{
Influence of a nonradiative reservoir on polariton spin multistability
}

\author{
M. Wouters, ${ }^{1, *}$ T. K. Paraïso, ${ }^{2, \dagger}$ Y. Léger, ${ }^{2}$ R. Cerna, ${ }^{2}$ F. Morier-Genoud, ${ }^{2}$ M. T. Portella-Oberli, ${ }^{2}$ and B. Deveaud-Plédran ${ }^{2}$ \\ ${ }^{1}$ Theory of Quantum and Complex Systems, Universiteit Antwerpen, B-2610 Antwerpen, Belgium \\ ${ }^{2}$ Laboratory of Quantum Optoelectronics, EPFL, CH-1015, Lausanne, Switzerland
}

(Received 15 February 2012; revised manuscript received 2 October 2012; published 4 January 2013)

\begin{abstract}
In this work, we study the influence of the excitation conditions on power-dependent spin switching and spin multistability of exciton polaritons in planar semiconductor microcavities. We obtain experimental evidence for the influence of a reservoir of nonradiative states which make a determining contribution to the dynamics of polaritons. While the spinor Gross-Pitaevskii equation (SGPE) fails in reproducing some critical experimental trends, an extended set of equations including a nonradiative reservoir allows us to reproduce the experiments quantitatively. We find that the energy renormalization of the exciton field due to the reservoir is crucial to describe power-dependent spin switching. The reservoir is also responsible for the effective repulsive interactions between polaritons of opposite spin obtained in the framework of the SGPE. Two important parameters, the coupling of the spinor polariton fields to the reservoir and the decay of the reservoir, are determined experimentally. We present indications that the reservoir originates from the formation of biexcitons and is constituted of localized exciton states.
\end{abstract}

DOI: 10.1103/PhysRevB.87.045303

PACS number(s): 71.36.+c

\section{INTRODUCTION}

Since its prediction by N. A. Gippius et al. ${ }^{1}$ in 2007 , polariton spin multistability rapidly appeared as a very promising phenomenon to realize spin-based optoelectronics devices with microcavity polaritons. Several proposals have shown the exceptional potential of this effect to realize spin memories $^{2}$ and polariton spintronic circuits. ${ }^{3}$ In a recent work, we demonstrated experimentally spin multistability with a single macroscopically occupied polariton level in a microcavity with patterned traps. ${ }^{4}$ Moreover, we evidenced the spin trigger effect, which is the spin analog for the Schmitt trigger in electronics, showing the possibility of developing advanced devices by simply using the intrinsic properties of microcavity polaritons. The potential of polaritons in spintronics is confirmed by other experimental achievements like the observation of spin rings $s^{5,6}$ and the polarization control of bistability propagation. ${ }^{7}$

The physics of spin multistability teaches us a lot about polariton spinor interactions. Indeed this phenomenon can only be observed in the presence of asymmetric spinor interactions. Because of that, spin-up and spin-down polaritons exhibit decoupled optical bistability curves. This leads to powerdependent spin switching and to spin multistability. Whereas the asymmetry of the spinor interactions is well accepted, the strengths of these interactions are still under intensive debate. In particular, the interactions between polaritons of opposite spins, which are expected to be attractive, have been suggested to be positive in several experiments. ${ }^{4,8}$ Moreover, we have shown that although the spinor Gross-Pitaevskii equations (SGPEs) are very efficient in reproducing qualitatively most of the features of spin multistability, they fail in meeting a satisfactory qualitative agreement. ${ }^{4}$

In the present work, we performed an exhaustive series of experiments in order to determine the behavior of multistability as a function of the different excitation parameters and, particularly, the cavity detuning. In agreement with recent propositions, ${ }^{6,9,10}$ we obtain strong indications that an important contribution to the dynamics comes from nonradiative states that are mostly populated in the presence of polaritons of opposite spins. We propose a theory going beyond the SGPE, including a reservoir dynamics. We obtain a good quantitative agreement with our experiments.

The paper is organized as follows. In Sec. II we introduce the phenomenology of polariton spin multistability based on experimental evidence. In Sec. III we model our experiments in the framework of the SGPE. We discuss the spinor interactions based on the experimental data. We highlight the crucial role of density-dependent nonlinear losses in the presence of polaritons of opposite spins, creating nonradiative states. We also show the limitations of the model in quantitative reproduction of the experiments (intensity jumps, narrowing of bistability width). In Sec. IV, we develop a more general theory in order to take into account the many-body effects and the energy renormalization due to the nonradiative reservoir. We show how this backaction is responsible for the effective repulsive interactions between polaritons of opposite spins. We successfully test the agreement of this theory with a series of experiments. In Sec. V, we discuss several physical issues of our theory with an emphasis on our understanding of the origin and role of the nonradiative reservoir. In Sec. VI, we describe theoretically the conditions for observing polarization multistability. Finally, we give a general conclusion to our work in Sec. VII.

\section{PHENOMENOLOGY OF SPIN MULTISTABILITY}

The first investigations of multistability with spinor systems were done in the early 1980s, with $\mathrm{Na}$ atom vapors showing a Zeeman splitting in the ground-state (GS) level. ${ }^{11,12}$ However, from the 1990s on, the research in this field dwindled, probably because no equivalent could be achieved in solid-state systems. The main difficulty in observing spin multistability is that it requires both the control of spinor interactions in the system and a strong spin coherence. The emergence of spintronics stimulated its theoretical investigation in different solidstate systems like ring cavities, ${ }^{13}$ magneto-optic cavities, ${ }^{14}$ spatial solitons in fibers, ${ }^{15}$ and, more recently, microcavity polaritons. . $^{1,3,16}$ 
(a)
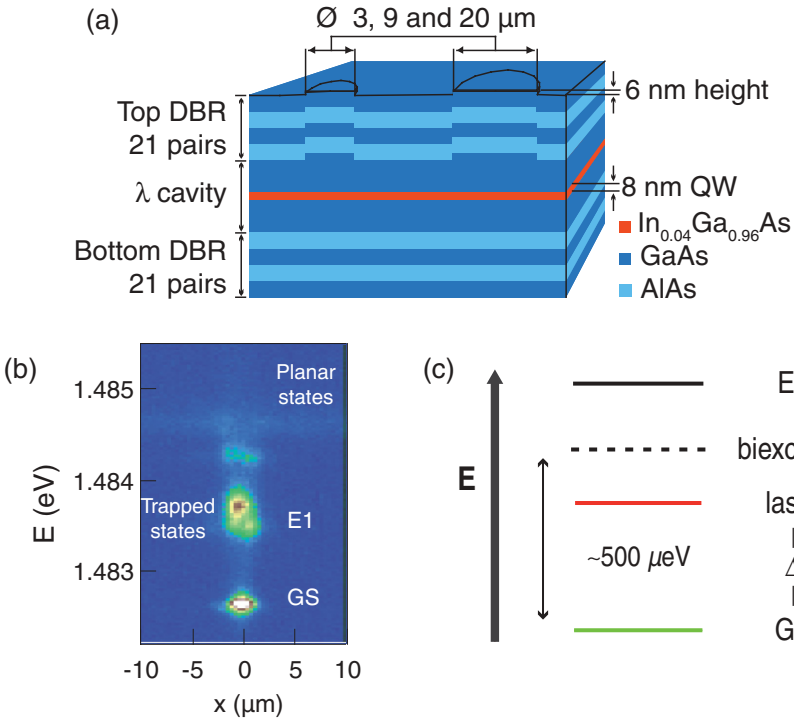

(c)

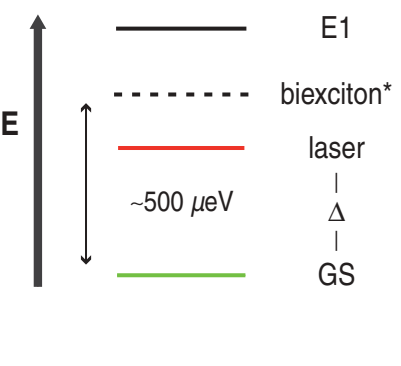

FIG. 1. (Color online) (a) Microcavity sample and mesa structures. The sample is wedged to tune the cavity length. ${ }^{18}$ (b) Spatially resolved polariton spectrum in a $3-\mu \mathrm{m}$ mesa. (c) Experimental conditions: we excite the ground state (GS) near-resonantly. We select a GS energy and a $\Delta$ such that the laser energy is close to the biexciton resonance. The first excited state E1 is about $1 \mathrm{meV}$ above the GS and its population is negligible.

The great potentialities of polaritons arise from the fact that they are light-matter quasiparticles with a very light effective mass and a de Broglie wavelength of a few microns. Polaritons inherit excellent coherence properties from their photonic nature, while their electronic nature favors significant spinor interactions in the nonlinear regime. ${ }^{17}$ In addition, quantum confinement can be achieved in micrometer-sized structures, ${ }^{18}$ and the coherent optical control of trapped polariton wave functions can be realized via simple optical means. ${ }^{19}$

Polariton optical bistability has been widely studied over the last 15 years. ${ }^{20-24}$ In this section, we show that spinor interactions strongly modify optical bistability. We first show how this can lead to high-efficiency spin switching. Then we give the experimental evidence of spin multistability. ${ }^{4}$

Our sample is a GaAs/AlAs semiconductor microcavity with an embedded $\operatorname{In}_{0.04} \mathrm{Ga}_{0.96}$ As quantum well [Fig. 1(a)]. Polaritons are trapped in patterned mesa structures engineered on top of the cavity spacer. ${ }^{18}$ In $3-\mu \mathrm{m}$ mesas, quantum confinement yields spectral discretization strong enough to allow us to work with single energy levels. The typical polariton line width $\gamma$ is $100 \mu \mathrm{eV}$ and the energy spacing between the first excited state (E1) and the GS can reach $1 \mathrm{meV}$ [Fig. 1(b)]. The mesa is excited using a single-mode continuous wave laser at normal incidence in the transmission configuration. We tune spinor interactions by adjusting the laser circular polarization degree $\rho_{\text {pump }}$ and power $P$ and detuning $\Delta$ from the GS. We also take advantage of the biexciton resonance to enhance nonlinearities [Fig. 1(c)].

Owing to selection rules on the total angular momentum, only polaritons with spin \pm 1 can be created by the cavity mode. ${ }^{16}$ Polaritons are thus properly described using the $\frac{1}{2}$-pseudospin formalism. Their spin state can be mapped in the Bloch sphere and is in one-to-one correspondence with

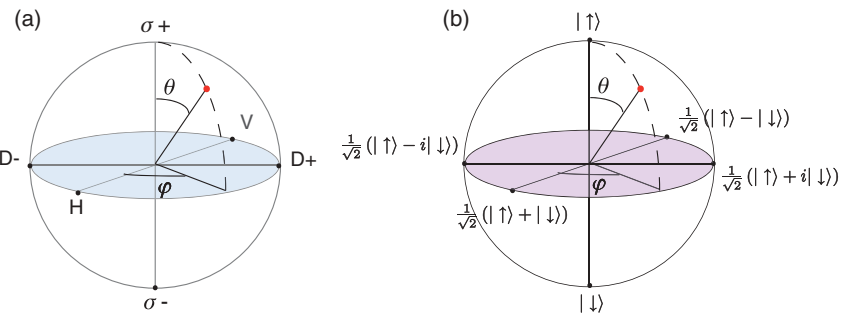

FIG. 2. (Color online) (a) Poincaré sphere. The right and left circularly polarized states are located at the north and south poles of the sphere, respectively. Linearly polarized states are on the equator. The other points of the sphere correspond to elliptically polarized states. (b) Bloch sphere. The $|\uparrow\rangle$ and $|\downarrow\rangle$ states are represented at the poles of the sphere. The states on the equator correspond to states with spin oriented in the $(x, y)$ plane.

the light polarization state on the Poincaré sphere (see Fig. 2). Right or left circularly polarized light $(\sigma+$ or $\sigma-)$ createsand is emitted by-spin-up $(|\uparrow\rangle)$ or spin-down $(|\downarrow\rangle)$ polaritons, respectively. Linearly polarized light creates polaritons in a coherent superposition of these spin states $|\uparrow \downarrow\rangle$ such as $|\uparrow \downarrow\rangle=\frac{1}{\sqrt{2}}\left(|\uparrow\rangle+e^{i \varphi}|\downarrow\rangle\right)$, where $\varphi$ is a phase factor. ${ }^{16}$

\section{A. Polariton spinor bistability}

Conventional optical bistability is obtained in our system under circularly polarized excitation. In Fig. 3(a), we show a bistability curve obtained experimentally with pure $\sigma+$ excitation. We measure the emission intensity as a function of the excitation power. When increasing the excitation power, the spin-up polariton density increases almost linearly up to a threshold power (upper threshold) where it jumps to a high-intensity branch. When decreasing the excitation power, spin-up polaritons remain on the high-intensity branch, describing a hysteresis loop, until another threshold is reached (lower threshold). Because $\sigma+$ excitation creates only spin-up polaritons, we observe a bistability cycle for spin-up polaritons only and almost no signal for spin-down polaritons. The internal polariton density is dominated by spin-up polaritons, and thus the transmitted light polarization is $\sigma+$ during the whole experiment [see Fig. 3(e)].

The experiments under elliptical excitation are displayed in Figs. 3(b) to 3(d). They reveal a much more complex phenomenology, with an interplay between the bistability cycles of spin-up and spin-down populations. The main features of this spinor bistability are the following.

(i) The upper bistability thresholds of both polariton spin populations coincide even when the excitation polarization is not completely linear, showing that the blue shift of one spin population induces the blue shift of the other spin population [Figs. 3(c) and 3(d)].

(ii) When the minority spin population jumps up, the majority spin population jumps down to an intermediateintensity bistability branch, whose intensity can be down to half the intensity of the upper branch [Fig. 3(b)].

(iii) The hysteresis of the minority population is inhibited by the density of the majority population, even close to linearly polarized excitation. This gives rise to an independence of the lower bistability thresholds, which can be significant even under linearly polarized excitation. These observations suggest 

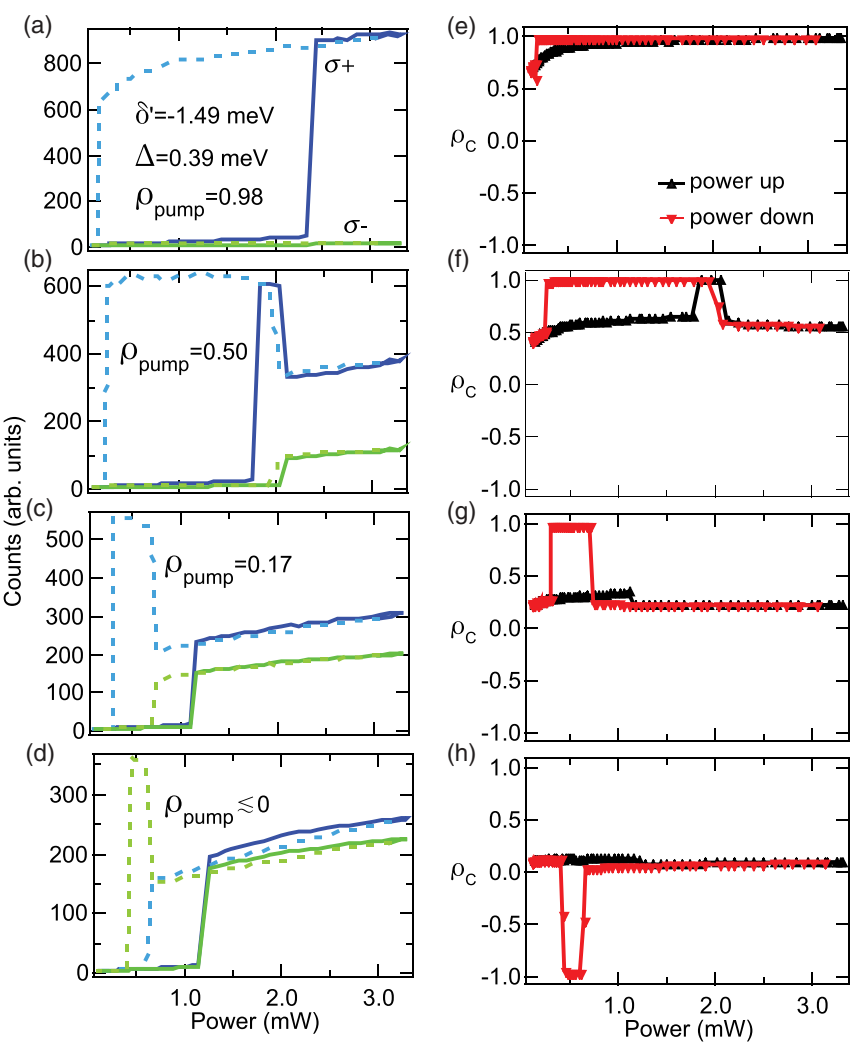

FIG. 3. (Color online) (a)-(d) Spinor bistability. Number of polaritons in the different polarizations when the pump power is ramped up (solid line) and down (dashed line) for different polarizations of the pump laser. (e)-(h) Power-dependent spin switch. Polarization of the polaritons along the power scan. The experiment was done at a position where the polariton energy was $\delta^{\prime}=-1.49 \mathrm{meV}$, relative to the exciton energy. The laser detuning was $\Delta=0.39 \mathrm{meV}$.

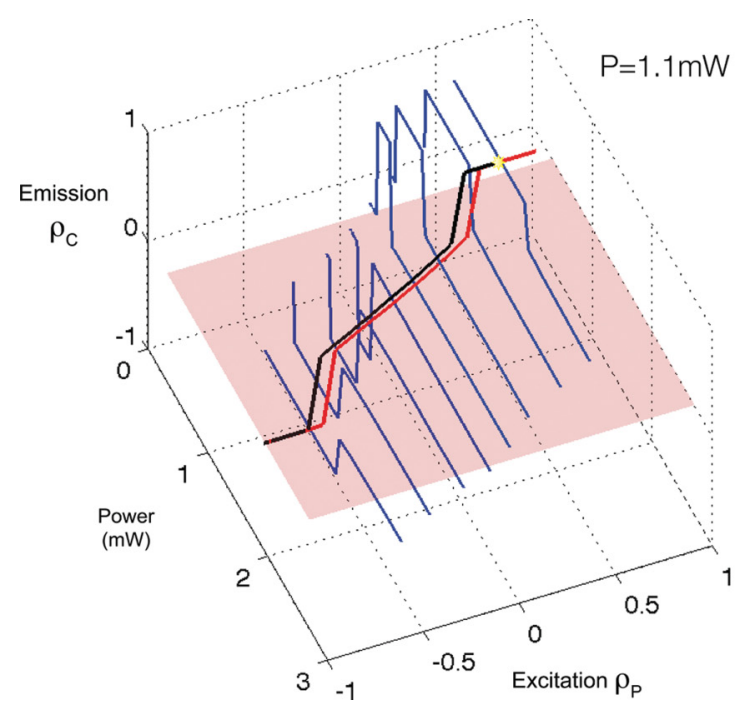

that important loss mechanisms occur in the presence of polaritons of opposite spins.

(iv) The upper thresholds occur at lower powers under linear excitation polarization than under circular polarization. This suggests that interactions between countercircular polaritons could be stronger than those between cocircular polaritons, in opposition to theoretical predictions. Actually, we will show that the lower threshold under linear polarization is rather a signature of a nonradiative contribution to the dynamics of polaritons.

\section{B. Polarization switching}

It is possible to calculate the emission polarization degree $\rho_{C}$ from the data in Figs. 3(a) to 3(d) using the relation

$$
\rho_{C}=\frac{I_{\sigma+}-I_{\sigma-}}{I_{\sigma+}+I_{\sigma-}},
$$

where $I_{\sigma+}$ and $I_{\sigma-}$ are the emission intensities from spin-up and spin-down polaritons, respectively. In Figs. 3(e) to 3(h), we show the circular polarization degrees versus the excitation power. In the forward direction, the emission polarization degree $\rho_{C}$ is close to the excitation polarization degree $\rho_{\text {pump }}$. In the case of $\sigma+$ excitation, $\rho_{C}=1$ during the whole scan (forward and backward) of the excitation power. Note that the discrepancy at very low power $(<500 \mu \mathrm{W})$ is an experimental artifact due to the very low signal-to-noise ratio of the transmission at such powers.

The independence of the bistability thresholds of spin-up and spin-down polaritons induces drastic changes in the spin polarization. In Fig. 3(f), when the excitation power is increased, spin-up polaritons jump to the high-intensity branch at $P=1.7 \mathrm{~mW}$, causing a switch of $\rho_{C}$ from 0.5 to 1. $\rho_{C}$ switches back to 0.5 at $P=2 \mathrm{~mW}$, where spin-down polaritons reach their upper threshold. When the excitation

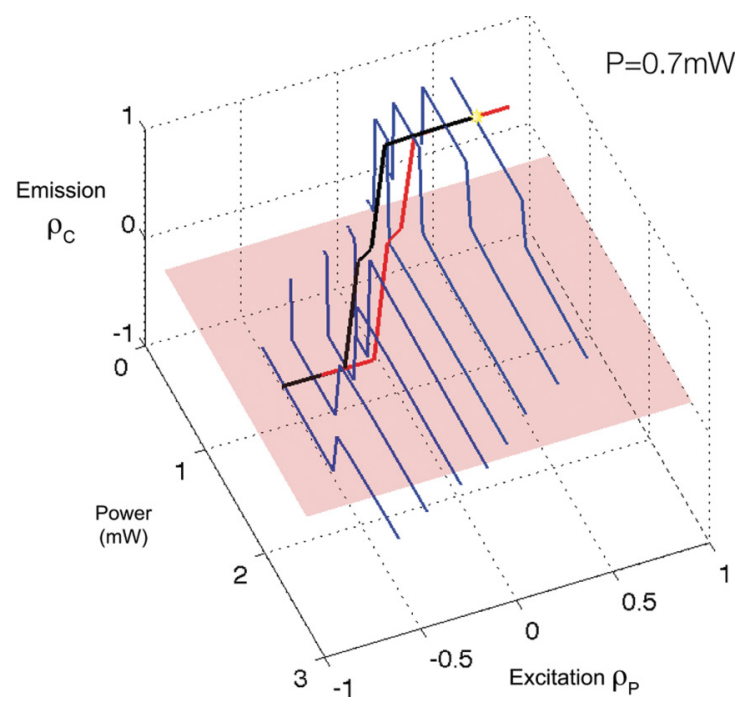

FIG. 4. (Color online) Principle of spin multistability. Blue curves display $\rho_{\mathrm{C}}$ vs $P$ for a fixed $\rho_{\text {pump }}$ obtained during the power-dependent spin switch experiment, when $P$ is decreased and a fully circularly polarized plateau appears between the lower bistability thresholds [see red curves in Figs. 3(e) to 3(h)]. Polaritons are initially prepared on a $\rho_{\mathrm{C}}=+1$ plateau at a given value of the excitation power [filled (yellow) circle]. We then look at the evolution of $\rho_{\mathrm{C}}$ when $\rho_{\text {pump }}$ is scanned backward (black curves) and forward (red curves). Depending on the excitation power, this produces either two distinct polarization hystereses (a) or two intersecting polarization hystereses (b). In the intersection region, $\rho_{\mathrm{C}}=-1,0$, and +1 are three stable states of the system. 
power is decreased, the independence of the lower thresholds (between 2 and $0.25 \mathrm{~mW}$ ) also causes a switch of $\rho_{C}$ to 1 .

For excitation polarizations closer to linear polarization, the upper thresholds of spin-up and spin-down polaritons are no longer independent. The switch occurs only between their lower thresholds, as shown in Figs. 3(g) and 3(h). Between the lower thresholds, the emission is characterized by a fully circularly polarized plateau $\left(\left|\rho_{C}\right|=1\right)$, denoting that the polariton spin population inside the microcavity is fully polarized. The size of the $\left|\rho_{C}\right|=1$ plateaus decreases as a function of $\left|\rho_{\text {pump }}\right|$, while their polarity depends on the sign of $\rho_{\text {pump. }}$. The system thus behaves as a high-contrast power-dependent spin switch.

\section{Spin multistability}

At a constant excitation power, the switch in polariton spin state is controlled by the excitation polarization degree. Multistability arises from the presence of robust polarization hysteresis upon back-and-forth scan of $\rho_{\text {pump }}$.

The system is first prepared in a fully polarized state. This is done either by using an elliptically polarized excitation and driving the power-dependent spin switch until it reaches a fully circularly polarized plateau (fcp) or simply by using a circularly polarized excitation. The polarization is then scanned, while the excitation power is maintained constant. Depending on the excitation power, the system exhibits either two distinct polarization hysteresis curves [see Fig. 4(a)] or a multistability cycle, i.e., two merged polarization curves [Fig. 4(b)].

In the case of the multistability cycle, the system admits three stable spin states for a given excitation condition $(P$, $\left.\rho_{\text {pump }}, \Delta\right)$ [Fig. 5(a)]. When the laser frequency corresponds to the biexciton resonance, the threshold independence is enhanced. In that particular case, the system can switch directly from $|\uparrow\rangle$ to $|\downarrow\rangle$ and hence behave as a spin Schmitt trigger [Fig. 5(b)]. The specifications of this multistable system such as its width and central polarization can be tuned by changing the excitation parameters.

\section{Multistability symmetry}

The symmetry of the multistability cycle strongly depends on the direction of the excitation linear polarization axis. By

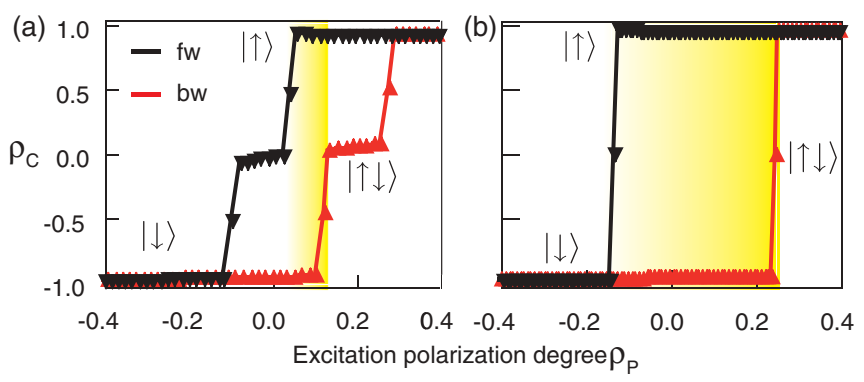

FIG. 5. (Color online) (a) Multistability arises from hysteresis in polarization-controlled spin switching at a constant excitation power. In dashed areas, three spin states are available for a given excitation condition (circles), enabling multivalued logic operations. (b) For a laser frequency at the biexciton resonance, the system behaves as a Schmitt trigger for spins.
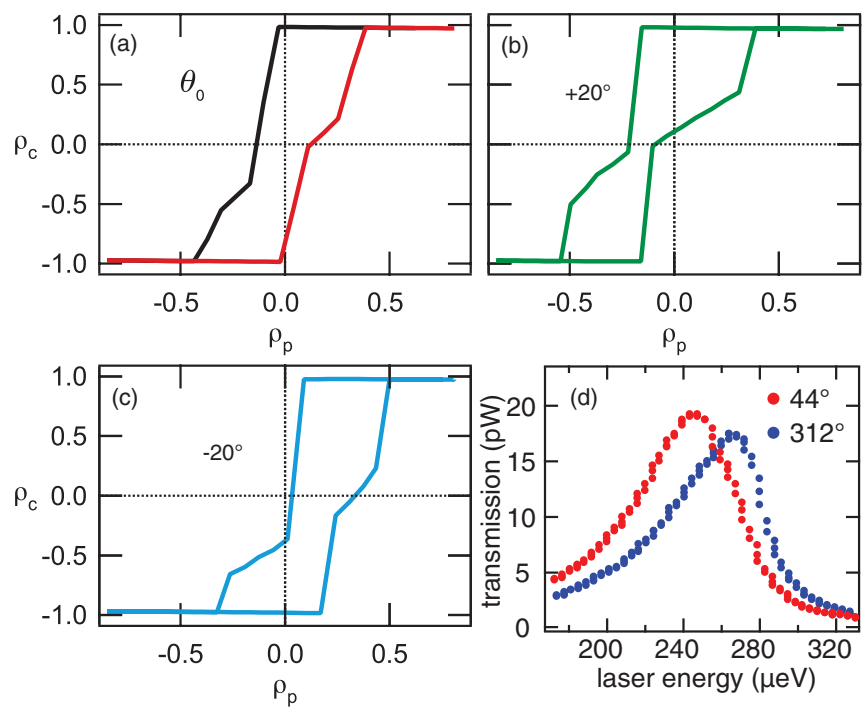

FIG. 6. (Color online) Symmetry. By rotating the linear polarization axis of the excitation, the multistability cycle can be made strongly asymmetric. The widths of the polarization hystereses are also modified. (a)-(c) Angles are indicated with respect to the reference orientation $\theta_{0}$, which corresponds to an orientation along D+, i.e., along one linear polarization splitting axis of the sample. (d) Polarization-resolved transmission spectrum in $3-\mu \mathrm{m}$ mesas. The excitation linear polarization axis was rotated with respect to our reference horizontal polarization axis, in steps of $4^{\circ}$. For each orientation, we scanned the laser energy over $70 \mathrm{GHz}$ at steps of $0.5 \mathrm{GHz}$. We obtained a linear polarization splitting of about $20 \mu \mathrm{eV}$, with orthogonal axes at $\pm 45^{\circ}$ with respect to our reference horizontal polarization axis. These axes correspond to the [001] (D+) and [010] (D-) crystallographic axes of our microcavity sample.

rotating the linear polarization direction of the excitation, it is possible to displace the position of the multistability region and to tune the asymmetry in the widths of the polarization hystereses. This is illustrated in Fig. 6. The most symmetric case [Fig. 6(a)] corresponds to a linear polarization close to the D+ axis, coinciding with one axis of a small linear polarization splitting observed in our sample. In Figs. 6(b) and 6(c) the multistability center is displaced to the left and to the right, respectively. Experimentally, the multistability behavior can hardly be achieved when the excitation polarization is exactly along the direction of the linear polarization splitting axes. The multistability width then becomes very small and bistability is easily lost. As we show below, the introduction of a linear polarization splitting in the theory is needed to reproduce these asymmetric multistability cycles. Simulations also confirm that multistability is more difficult to achieve close to the polarization splitting axes.

\section{SPINOR GROSS-PITAEVSKII EQUATION}

In this section we discuss the description of our experiments based on the SGPEs. We define two polariton fields, $\psi_{\uparrow, \downarrow}$, for spin-up and spin-down polaritons, respectively, that are coupled to each other by spinor interactions. The main characteristic of this model is the anisotropy of polariton spinor interactions, i.e., the fact that the interaction strengths between polaritons of same $\left(\alpha_{1}\right)$ or opposite $\left(\alpha_{2}\right)$ spin are different. 
One can understand the origins of this anisotropy through a microscopic analysis of polariton-polariton interaction. It has been shown that because of excitons' composite nature, carrier exchange processes are the dominant scattering mechanisms. ${ }^{25}$ The strong dependence of the exchange interactions on the spin of the carriers involved is reflected in the anisotropy of polariton spinor interactions. The blue shifts of spin-up and spin-down polaritons are thus given by $\Delta \epsilon_{\uparrow}=\alpha_{1} n_{\uparrow}+\alpha_{2} n_{\downarrow}$ and $\Delta \epsilon_{\downarrow}=\alpha_{1} n_{\downarrow}+\alpha_{2} n_{\uparrow}$, respectively.

We consider the main features of the spinor bistability experiments (Sec. II A), which are the coincidence of the upper thresholds, the appearance of an intermediate-intensity branch due to nonlinear losses in the presence of polaritons of opposite spins, the independence of the lower thresholds, and the effect of linear polarization splitting. We first ignore the renormalization of the Hopfield factors and the contribution of nonradiative states to the polariton dynamics.

The two coupled equations for the field amplitudes $\psi_{ \pm}$in the $(\sigma+, \sigma-)$ basis read

$$
\begin{aligned}
i \frac{d}{d t} \psi_{\uparrow, \downarrow}= & {\left[-\Delta-\frac{i}{2}\left(\gamma+\beta\left|\psi_{\downarrow, \uparrow}\right|^{2}\right)+\alpha_{1}\left|\psi_{\uparrow, \downarrow}\right|^{2}\right.} \\
& \left.+\alpha_{2}\left|\psi_{\downarrow, \uparrow}\right|^{2}\right] \psi_{\uparrow, \downarrow}+\frac{\epsilon_{\operatorname{lin}} \psi_{\downarrow, \uparrow}}{2}+F_{\uparrow, \downarrow},
\end{aligned}
$$

where $F_{\uparrow, \downarrow}$ is the excitation field, $\gamma$ the polariton line width, and $\epsilon_{\text {lin }}$ the strength of the linear polarization splitting. The intensity losses observed in the presence of polaritons with opposite spins are modeled by the introduction of a nonlinear contribution to the decay rates through the parameter $\beta$.

\section{A. Comparison with experiments}

The results of the theoretical investigations using the SGPE are summarized in Fig. 7. In Figs. 7(a) to 7(d), we model the experimental trends in Figs. 3(a) to 3(d). The qualitative agreement is very satisfactory. Under circularly polarized excitation, the conventional bistability is obtained [Fig. 7(a)]. Under elliptically polarized excitation, both spin-up and spin-down populations exhibit bistability. The bistability hysteresis of the minor population is inhibited and an intermediate-intensity branch is formed in the presence of both spin populations. In Fig. 7(b), $\rho_{\text {pump }}=0.5$, the upper thresholds are different and a significant loss of intensity is observed for spin-up when spindown polaritons reach their upper threshold. As in Fig. 3(b), the bistability width of spin-down polaritons is very narrow. At $\rho_{\text {pump }}=0.17$ [Fig. 7(c)], the upper thresholds coincide and the lower thresholds remain different. The independence of the lower thresholds under linearly polarized excitation is also reproduced. In Fig. 7(d), we use $\rho_{\text {pump }}=-0.001$ to highlight the system's sensitivity to the sign of $\rho_{\text {pump }}$.

In the framework of the SGPE, exciton-photon detuning is not taken into account. Hence, it is important to note that the values of the interaction constants $\alpha_{1}, \alpha_{2}$, and $\beta$ are effective values that should be adjusted according to the polariton GS energy. We chose to scale $\alpha_{2}$ and $\beta$ as functions of $\alpha_{1}$. In the case of Fig. 7, the parameters are $\Delta=0.39 \mathrm{meV}, \gamma=$ $0.05 \mathrm{meV}, \alpha_{2}=0.33 \alpha_{1}$, and $\beta \simeq 0.2 \alpha_{1}$, while the value of $\alpha_{1}$ is arbitrary in our mean-field theory, because it just leads to a rescaling of the laser power.
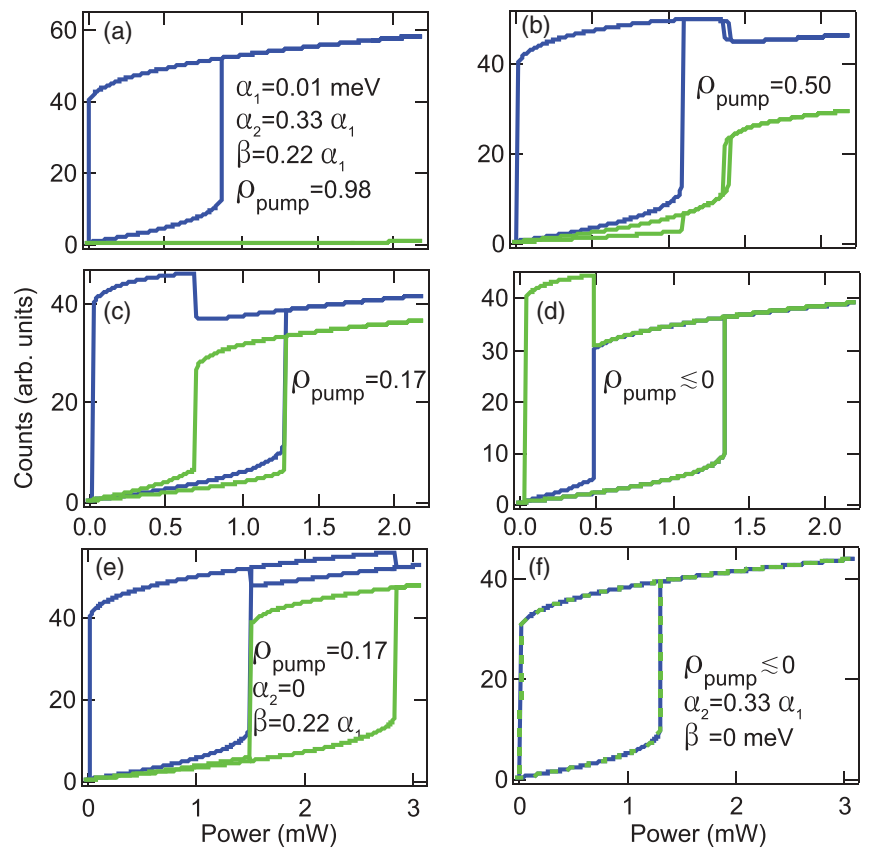

FIG. 7. (Color online) Spinor bistability (SGPE) theoretical simulations. (a)-(d) Qualitative reproductions of the experimental observations in Figs. 3(a)-3(d). (e) Illustration that a nonzero $\alpha_{2}$ is needed; (f) the necessity of a nonzero $\beta$.

\section{Role of $\alpha_{2}$ and $\beta$}

We are first concerned with modeling the coincidence of the upper bistability thresholds under elliptically polarized excitation. As explained previously, the upper thresholds can coincide only if the increase in one spin population causes the blue shift of the other. Therefore we need to use a positive $\alpha_{2}$ in Eq. (1). This means that in order to model spinor bistability in the framework of the SGPE we need to consider repulsive effective interactions between polaritons of opposite spins. Several effects, like the onset of saturation of strong coupling, the proximity to the biexciton resonance, and the additional blues shift due to nonradiative states, may contribute to an effective $\alpha_{2}>0$. We discuss this issue in Sec. V. As an example, we show in Fig. 7(e) that with $\alpha_{2} \leqslant 0$ it is not possible to obtain the same upper thresholds under elliptically polarized excitation. Increasing $\alpha_{2}$ brings the upper threshold of spin-down polaritons closer to that of spin-up polaritons.

The importance of nonlinear losses is shown in Fig. 7(f). We show that in the case of $\alpha_{2}>0$ and $\beta=0$, the lower thresholds also tend to be the same for spin-up and spin-down polaritons close to linearly polarized excitation. In fact, in the absence of any nonlinear loss mechanism, repulsive interactions will tend to equalize the bistability thresholds, and the coincidence of lower thresholds occurs for smaller $\alpha_{2}$ than the coincidence of upper thresholds.

Another important issue is the formation of an intermediateintensity branch in the presence of both spin populations. This phenomenon can only be reproduced by the introduction of nonlinear losses.

To summarize, the coincidence of spin-up and spindown bistability thresholds can only be reproduced with effective repulsive interactions $\left(\alpha_{2}>0\right)$. In order to recover 

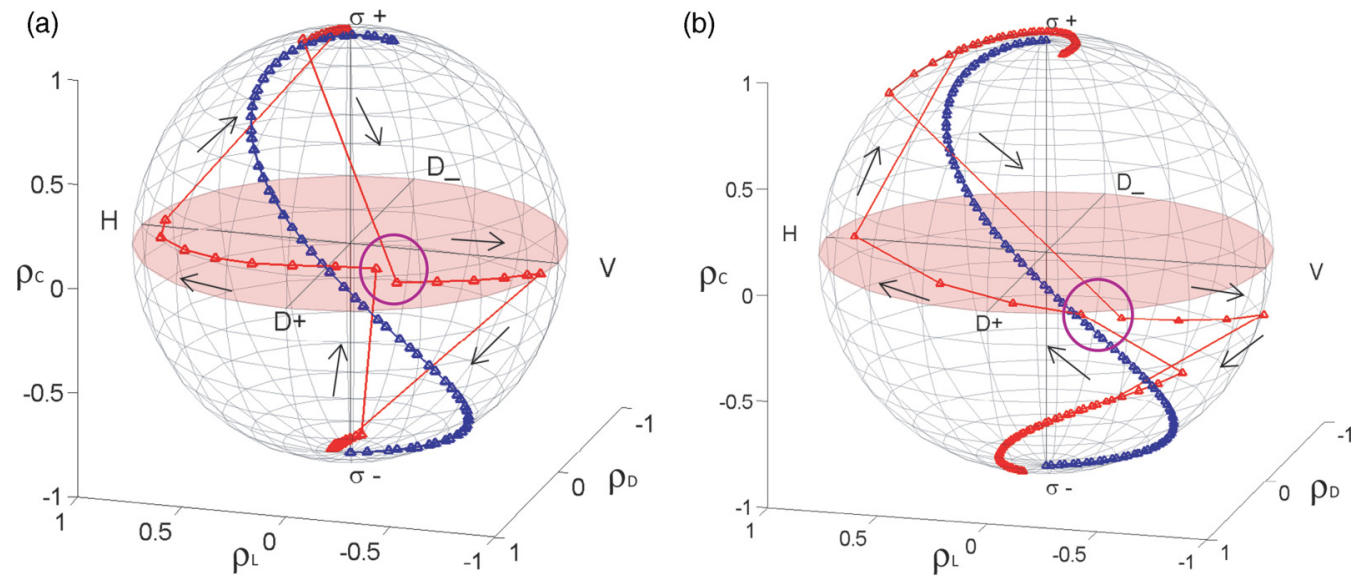

FIG. 8. (Color online) Poincaré sphere in the multistability regime. (a) Experimental data presented in Fig. 5. (b) Theory. The experimental features of spin multistability are well reproduced: the jumps, the multistability gap, the pseudospin precession, and the conservation of coherence. The pseudospin trajectory (red triangles) is clearly distinct from the trajectory of the excitation (blue triangles). Coherence is maintained $(|\rho| \simeq 1)$ during the whole cycle.

independent lower thresholds and to reproduce the formation of an intermediate-intensity branch, it is necessary to consider nonlinear contributions to the polariton decay rates $(\beta>0)$.

Let us now compare our findings with the original prediction by Gippius, made in the ideal case of noninteracting spinup and spin-down populations ( $\alpha_{2}=0$ and $\beta=0$ ). In this case, multistability can be achieved as soon as $P_{\text {low }} / 2<P<P_{\text {up }} / 2$ ( $P_{\text {up }}$ and $P_{\text {low }}$ are the lower and upper bistability thresholds, respectively). Our experiments show that it is not possible to treat spin-up and spin-down independently. In addition, the achievement of the spin trigger regime is a direct consequence of the presence of nonlinear losses and we were not able to simulate it without them.

\section{Macroscopic spin coherence}

The coherence of spin-up and spin-down polaritons was unambiguously evidenced by measuring the pseudospin trajectory in the Poincaré sphere during a multistability cycle. We resolved emission polarization along the three axes of the Poincaré sphere in order to access the three Stokes parameters, i.e., the emission polarization degrees in the circular $\rho_{\mathrm{C}}$, linear $\rho_{\mathrm{L}}$, and diagonal $\rho_{\mathrm{D}}$ bases, given by

$$
\rho_{\mathrm{C}}=\frac{I_{\sigma+}-I_{\sigma-}}{I_{\sigma+}+I_{\sigma-}}, \quad \rho_{\mathrm{L}}=\frac{I_{\mathrm{H}}-I_{\mathrm{V}}}{I_{\mathrm{H}}+I_{\mathrm{V}}}, \quad \rho_{\mathrm{D}}=\frac{I_{\mathrm{D}+}-I_{\mathrm{D}-}}{I_{\mathrm{D}+}+I_{\mathrm{D}-}} .
$$

The experimental results are shown in Fig. 8(a). The vertical position corresponds to the circular polarization degree. The azimuthal angle $\varphi$ on the sphere corresponds to the phase difference between spin-up and spin-down polaritons. It depends on the linear and diagonal polarization degrees:

$$
\varphi=\arctan \frac{\rho_{\mathrm{D}}}{\rho_{\mathrm{L}}} .
$$

The inclination angle $\theta$ is related to the ellipticity of light and is given by

$$
\theta=\arccos \frac{\rho_{\mathrm{C}}}{\sqrt{\rho_{\mathrm{D}}^{2}+\rho_{\mathrm{L}}^{2}}} .
$$

The norm of the pseudospin vector is a measurement of the spin coherence

$$
|\rho|=\sqrt{\rho_{\mathrm{C}}^{2}+\rho_{\mathrm{L}}^{2}+\rho_{\mathrm{D}}^{2}} \leqslant 1 .
$$

The experiment starts on the $\sigma-$ pole of the sphere, where the pseudospin is pinned before jumping abruptly close to the $\mathrm{D}+$ state. A wide precession towards the $\mathrm{H}$ state is then observed before the second jump to the $\sigma+$ pole occurs. On the backward path, a third jump brings the pseudospin farther from the $\mathrm{D}+$ state, producing a gap in the precession trajectory. The pseudospin then precesses towards the $\mathrm{V}$ state and, finally, jumps back to the $\sigma-$ state. The trajectory of the excitation is displayed in Fig. 8(b) (blue triangles). It corresponds to the trajectory of a linearly polarized $(\mathrm{H})$ excitation beam transmitted through a quarter-wave plate. Clearly, the trajectories of the polariton and excitation pseudospins are strikingly different. The precession of the polariton pseudospin close to linearly polarized excitation is similar to that observed in polariton scattering experiments. ${ }^{17}$ It results from the excitation-induced splitting between spin-up and spin-down polariton states, which acts qualitatively as an effective magnetic field on polariton spins: When almost linearly polarized polaritons are injected into the microcavity, their spins start precessing to the left or to the right depending on the direction of this effective magnetic field along the pole axis. This direction is given by the sign of the excitation circular polarization rate. Obviously, the observed rotation depends on both the value of the excitation induced splitting and the polariton lifetime. We observe that the spin coherence is preserved during the whole multistability cycle, as expected for such a resonant experiment. In Fig. 8(b), we show that the experimental features are well reproduced by the spinor Gross-Pitaevskii model.

\section{Linear polarization splitting}

A final success of this model concerns the effect of the linear polarization splitting observed in our microcavity. Such a splitting will act as a second effective magnetic field on the polariton pseudospin. This new field lays in the equatorial 
plane of the Poincare sphere roughly in the direction of the $\mathrm{D}+$ axis (see Sec. IID). Because this linear polarization splitting is only a few tens of micro-electron volts (see Fig. 6) it has almost no effect when the excitation-induced splitting is large (when the pseudospin is close to the poles) but it contributes quite strongly to the dynamics when both polariton spin populations are in resonance with the laser (when the pseudospin is close the equator). In the latter case, the pseudospin trajectory rotates around the D+ axis, explaining the possible shift of the pseudospin from the equator towards more elliptical polarizations depending on the relative direction of the pseudospin trajectory compared to the D+ axis. This explains the results in Fig. 8.

\section{B. Limitations of the SGPE}

The SGPE model presented above reproduces successfully the main experimental observations and the phenomenology of spin multistability. However, this approach is not sufficient to meet quantitative agreement. In particular, the following experimental observations could not be reproduced in the calculations.

(i) The upper threshold is at lower power under linearly polarized excitation (see Fig. 3).

(ii) The intensity jumps in the spinor bistability are underestimated by the model (Figs. 3 and 5), and so are the ratio between the high- and the-intermediate intensity branches and the polarization degree near linear excitation during the multistability cycle (Fig. 7).

(iii) In the Bloch sphere experiment, the pseudospin vector remains closer to the poles than in theory (Fig. 8). This is a direct consequence of the previous point. Indeed, the described underestimation of the increase in the major population at the lower thresholds leads to an underestimation of the polarization degree.

To understand these limitations, it is important to recall that the SGPE model does not account for the energy shift induced by the nonradiative population.

The change in effective detuning when the polaritons blue shift is not modeled by the SGPE using polariton fields directly. Actually, when the polariton density is increased, the exciton energy should renormalize independently of the cavity photon's energy, leading to an effective change in the cavity detuning. The Hopfield factors thus change drastically at the bistability thresholds. We thus expect that the photonic Hopfield factor of the lower polaritons is larger on the upper branch, which leads to a higher emission intensity. This effect is simply taken into account in the theory by working directly in the exciton-photon basis.

A more profound shortcoming of model 1 is that the blue shift due to the nonradiative reservoir is ignored. We show in the next section that taking its effect into account does improve the quantitative agreement with experiments.

\section{EXTENDED THEORY}

\section{A. Evidence of a reservoir}

The SGPE theory gives a reasonable qualitative reproduction of the experimental observations. In Sec. III B, we have, however, pointed out the limitations of the model from the quantitative point of view. In this section, we show that taking into account the blue shift of the exciton level due to the interactions with a (bi-)excitonic reservoir substantially improves the accuracy of the theory.

The creation of an excitonic reservoir is indeed expected because of the long exciton lifetime in the absence of radiative coupling. In our SGPE model, we have introduced the nonlinear loss term, which was physically attributed to the formation of biexcitons. ${ }^{4}$ These biexcitons do not immediately disappear from the quantum well and can also scatter towards dark or high- $k$ exciton pairs. All these contributions cause a blue shift of the exciton level. To include this effect, we need to consider the following system of five equations:

$$
\begin{aligned}
i \frac{d}{d t} \chi_{\uparrow, \downarrow}= & {\left[-\varepsilon_{X}-\frac{i}{2}\left(\gamma_{X}+\beta\left|\chi_{\downarrow, \uparrow}\right|^{2}\right)+g_{R} n_{R}\right.} \\
& \left.+\alpha_{1}\left|\chi_{\uparrow, \downarrow}\right|^{2}+\alpha_{2}\left|\chi_{\downarrow, \uparrow}\right|^{2}\right] \chi_{\uparrow, \downarrow}+\frac{\Omega_{R}}{2} \varphi_{\uparrow, \downarrow}, \\
i \frac{d}{d t} \varphi_{\uparrow, \downarrow}= & {\left[-\varepsilon_{c}-\frac{i}{2} \gamma_{c}\right] \varphi_{\uparrow, \downarrow}+\frac{\Omega_{R}}{2} \chi_{\uparrow, \downarrow} } \\
& +\frac{\epsilon_{\text {lin }}}{2} \varphi_{\downarrow, \uparrow}+F_{\uparrow, \downarrow} e^{-i \omega_{L} t}, \\
\frac{d}{d t} n_{R}= & 2 \beta\left|\psi_{\uparrow}\right|^{2}\left|\psi_{\downarrow}\right|^{2}-\gamma_{R} n_{R},
\end{aligned}
$$

where $\chi(\varphi)$ is the exciton (photon) field with energy $\varepsilon_{X(c)}$ and decay rate $\gamma_{X(c)}, \Omega_{R}$ the Rabi splitting, $n_{R}$ the reservoir density, $g_{r}$ the exciton-reservoir interaction constant, and $\gamma_{R}$ the reservoir decay rate. All the other terms appeared in Eq. (1) and have been adjusted to the exciton-photon basis (we consider here that the linear polarization splitting has a photonic origin). The nonlinear feeding of the reservoir by pairing excitons of opposite spins is still modeled by the parameter $\beta$. The value of $g_{r}$ is assumed to be $\left[\alpha_{1}+\alpha_{2}\right] / 2$, which gives a good order of magnitude of the biexciton-exciton interaction and provides a repulsive interaction as $\alpha_{1} \gg \alpha_{2}$.

Linear contributions to the scattering of polaritons to the nonradiative reservoir such as phonon absorption ${ }^{9}$ are not included in the present model. If it is assumed that spin relaxation is slower than the decay rate, then a linear scattering to the reservoir can be effectively described by evenly renormalizing the polariton spinor interaction constants $\alpha_{1}$ and $\alpha_{2}$ by the same factor. In the opposite regime of fast spin relaxation, it was shown in Ref. 9 that this renormalization leads to an effective sign change of $\alpha_{2}$ from negative to positive and to the same conclusions as the SGPE, (1), with a positive $\alpha_{2}$. Therefore linear scattering processes to the reservoir are not sufficient to solve the limitations of the SGPE discussed previously.

The dependence of the upper bistability threshold on the excitation polarization is the most direct experimental evidence of the reservoir. Our SGPE predicts an increase in the power threshold by roughly a factor of two when going from circularly to linearly polarized excitation, because interactions between countercircularly polarized excitons are much weaker than interactions between cocircularly polarized excitons (see Fig. 7). In experiments, however, the upper bistability threshold under linear polarization can be much lower than under circular excitation. 

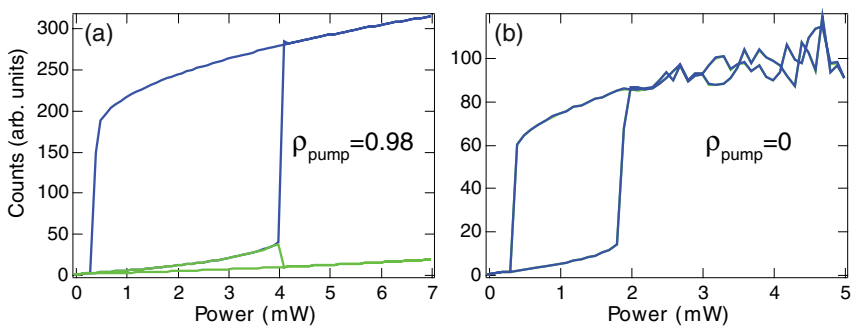

FIG. 9. (Color online) Illustration of the effect of interactions between the reservoir and the lower polariton branch on the upper thresholds. The upper threshold under linear excitation can decrease dramatically when these interactions are taken into account. In this example, the upper threshold under linear excitation (b) is a factor of two smaller than that under circular excitation (a). The reservoir interactions are also responsible for the suppression of the polariton density when both polariton spin polarizations are on the upper bistability branch (b). Parameters: $\Delta=0.22 \mathrm{meV}, \delta^{\prime}=$ $-1.96 \mathrm{meV}, \quad \gamma_{C}=0.05 \mathrm{meV}, \quad \gamma_{X}=0.05 \mathrm{meV}, \quad \alpha_{2} / \alpha_{1}=-0.15$, $\beta / \alpha_{1}=0.5$, and $\Omega_{R}=1.5 \mathrm{meV}$.

The role of the reservoir can be understood as follows. Under circular excitation, there is only one polariton polarization so the reservoir is not populated. As expected from conventional bistability, the upper bistability threshold increases with the laser-polariton detuning. Under elliptical and linear excitation, the reservoir becomes populated because of the presence of polaritons of opposite spins. The interaction with the reservoir brings the polariton levels closer to the laser frequency, which reduces the effective laser-polariton detuning. The upper threshold is lower than the SGPE prediction.

A quantitative illustration of the above is given in Fig. 9, where we show a simulation of the bistability under circular and linear polarization when taking into account the interactions between polaritons and the reservoir. Because of the blue shift due to the reservoir the upper bistability threshold is a factor of two smaller under linearly polarized excitation.

A second experimental feature that reflects the backaction of the reservoir on the polaritons is the suppression of the polariton density under linearly polarized excitation, when both polariton spin polarizations are on the upper branch. While the SGPE does show this feature qualitatively, the magnitude of the density suppression and jump is only of the order of $10 \%$. As also shown in Fig. 9, when the blue shift due to the reservoir is included, the experimentally observed suppression of more than a factor of two is reproduced.

The same effect is observed at fixed, weakly elliptical, excitation polarization, when the laser power is decreased and the minority polarization reaches its lower threshold. After it has fallen down, the majority density typically increases by a factor of two (see Fig. 3). We illustrate in Fig. 10 that the reservoir is needed to reproduce this effect theoretically. In Fig. 10(a), the polariton blue shift was modeled with an effective $\alpha_{2}>0$ and the jump is only of the order of $10 \%$. In Fig. 10(b), where the reservoir blue shift is included, the jump is of a factor two, as observed experimentally [Figs. 3(b)-3(d)]. In these simulations, we have used a negative value of $\alpha_{2}$, but in the presence of polariton-reservoir interactions, the sign of $\alpha_{2}$ only plays a minor role (see discussion below).
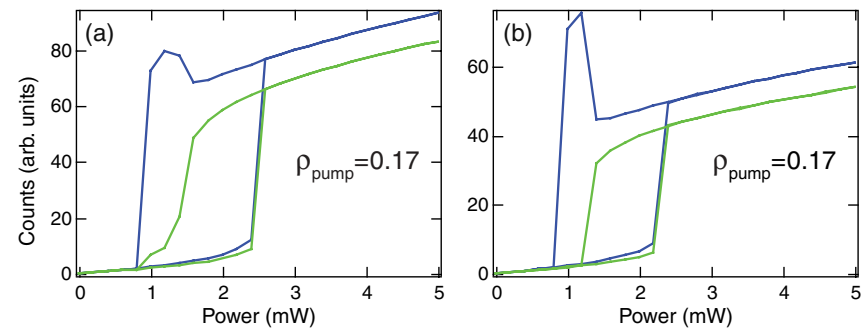

FIG. 10. (Color online) Illustration of the theoretical reproduction of the suppression of the polariton density when both spin polarizations are on the upper branch. (a) SGPE. (b) Blue shift of polaritons induced by the reservoir is crucial to reproduce this effect with the same strength as in the experiments. Same parameters as in Fig. 9.

The reason is that the polariton field is pushed away from resonance with the laser frequency due to the interaction with the reservoir, which leads to a density reduction. The magnitude of the density reduction reveals that the blue shift due to the reservoir is of the order of the (nonlinearly enhanced) polariton line width.

\section{B. Interpreting experimental detuning studies}

The quantitative reproduction of the experimental data with our extended model allows us to compare the variations of the fitting parameters with the exciton-photon detuning to theoretical expectations. This analysis provides a good indication of the model validity.

The two ingredients of our model that are expected to vary most with the exciton-photon detuning are the exciton line width $\gamma_{X}$ and the nonlinear loss rate $\beta$. The widening of the line width for increasing polariton energy is indeed expected due to the stronger interaction of polaritons with excitonic states feeling the excitonic disorder. One should thus consider an effective exciton line width, very broad when the polariton energy falls within the inhomogeneous exciton line width and much smaller when it lies below. ${ }^{26}$ The nonlinear loss rate, on the other hand, is expected to be maximal when the energy of the polaritons is at the biexciton resonance.

In Table I, we summarize how the fitting parameters vary with the polariton detuning with respect to the exciton energy $\delta^{\prime}=\epsilon_{p}-\epsilon_{X}$. Two trends are indeed obvious: the exciton line width increases monotonically with the laser-exciton detuning $\left(\hbar \omega_{L}-\epsilon_{X}=\delta^{\prime}+\Delta\right)$. The nonlinear loss rate $\beta$ increases when the laser frequency approaches the biexciton resonance.

\section{Large negative detuning}

Let us now present the comparison between experimental data and theoretical simulations in more detail. In Fig. 11, we show the experimental data and theoretical simulations at

TABLE I. Model parameters for the extended SPGE for various values of the polariton-exciton detuning $\delta^{\prime}$.

\begin{tabular}{lcccc}
\hline \hline & \multicolumn{4}{c}{$\delta^{\prime}$} \\
\cline { 2 - 5 } & $-2.48 \mathrm{meV}$ & $-1.71 \mathrm{meV}$ & $-1.49 \mathrm{meV}$ & $-1.2 \mathrm{meV}$ \\
\hline$\beta / \alpha_{1}$ & 0.05 & 0.5 & 0.5 & 2 \\
$\gamma_{X}(\mathrm{meV})$ & 0.01 & 0.05 & 0.05 & 0.3 \\
\hline \hline
\end{tabular}



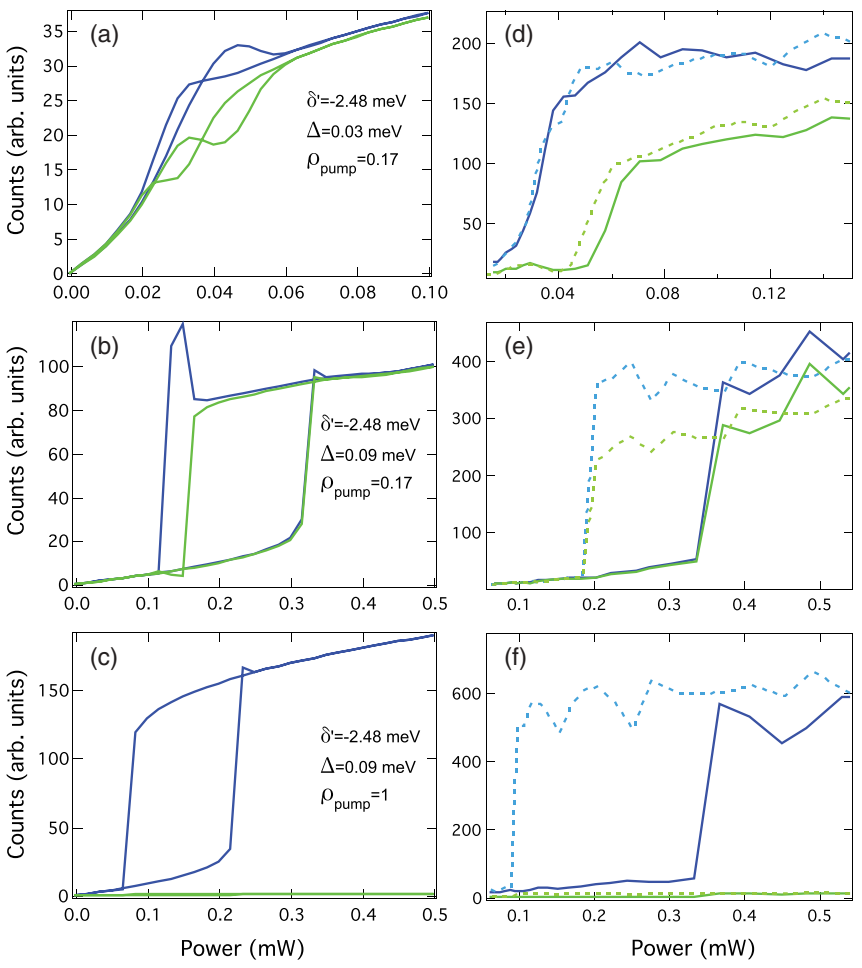

FIG. 11. (Color online) Comparison between theoretical (a)-(c) and experimental (d), (e) bistability curves at a large negative detuning, $\delta^{\prime}=-2.48 \mathrm{meV}$. (a), (d) For small laser-polariton detuning, $\Delta=$ $0.03 \mathrm{meV}$, separate thresholds are observed under almost linearly polarized excitation. A higher value of the detuning, $\Delta=0.09 \mathrm{meV}$, is considered for almost-linear (b), (e) and circular (c), (f) polarization. Simulation parameters: $\gamma_{C}=0.05 \mathrm{meV}, \gamma_{X}=0.01 \mathrm{meV}, \alpha_{2} / \alpha_{1}=$ $-0.15, \beta / \alpha_{1}=0.05, \Omega_{R}=1.5 \mathrm{meV}$.

a large negative detuning, where the lower polariton is far from the exciton energy and from the biexciton resonance. The experimental bistability is obtained for a very low value of the laser-polariton detuning, $\Delta=0.09 \mathrm{meV}$, indicating a very narrow effective polariton line width (the minimal value of the detuning to obtain bistability scales with the line width as $\left.\Delta_{\min }=\sqrt{3} \gamma / 2\right){ }^{20}$ At this critical detuning, the thresholds for the two polarizations to jump to the upper branch do not coincide [Figs. 11(a) and 11(d)]. Instead, when the dominant polarization jumps up, the other one is suppressed. This behavior is indicative of either an attraction between the two polarization components or nonlinear losses. The latter mechanism is, however, not compatible with the small hysteresis observed in the minority polarization. These experiments thus give an indication of attractive interactions between countercircularly polarized polaritons, $\alpha_{2}<0$.

At a laser-polariton detuning $\Delta=0.09 \mathrm{meV}$, the upper thresholds for both polarizations coincide [Figs. 11(b) and 11(e)]. As indicated above and also described in Ref. 6, the buildup of a reservoir can explain this feature. The simulations in Fig. 11(b) confirm this hypothesis. The reservoir blue shift is able to compensate for the attractive interactions between countercircularly polarized polaritons, so that both polarizations jump up simultaneously, as if $\alpha_{2}$ were positive.
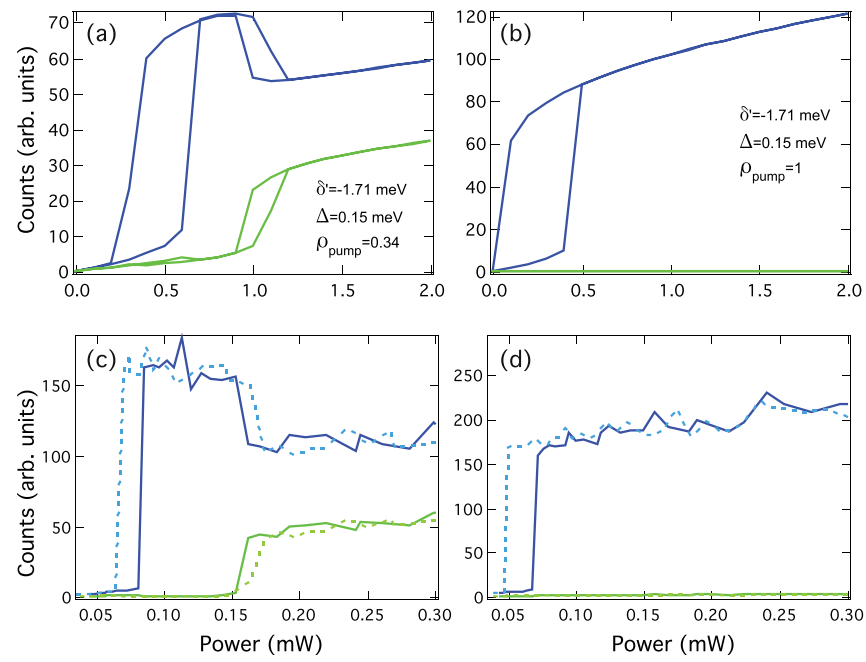

FIG. 12. (Color online) Comparison between experimental and theoretical bistability curves when the biexciton resonance is approached, $\delta^{\prime}=-1.71 \mathrm{meV}$. (a), (b) Theory. (c), (d) Experiment. Simulation parameters: $\gamma_{X}=0.05 \mathrm{meV}, \beta / \alpha_{1}=0.5$.

\section{Increasing detunings}

Upon increasing the cavity detuning, and thus the lower polariton energy, one should consider an increase in the excitonic line width, because strong coupling can no longer protect excitons against the structural disorder in the quantum wells. ${ }^{27}$ Consequently, even under circular polarization, the critical detuning for bistability increases. This feature can be reproduced in theoretical simulations by increasing the exciton line width (Figs. 12-14). Because the laser energy approaches the biexciton resonance, it is not surprising that the experimental observations require a larger value of $\beta$ for a satisfactory theoretical reproduction (see Table I).

The constant $g_{R}$ that gives the blue shift due to the reservoir is not expected to be changed. The simulations show, however, that a smaller value of $g_{R}$ is needed to obtain satisfactory
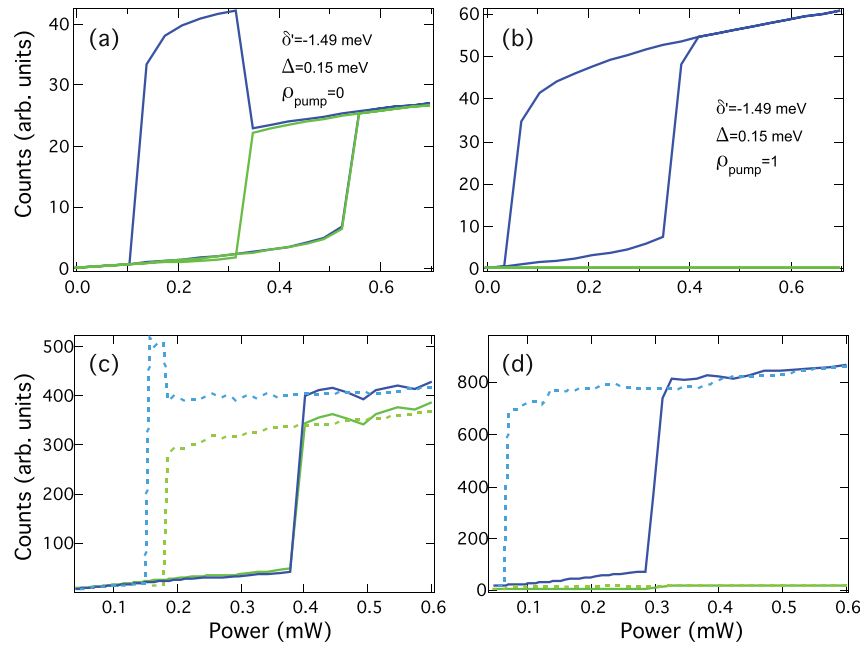

FIG. 13. (Color online) Comparison between experimental and theoretical bistability curves close to the biexciton resonance $\delta^{\prime}=$ $-1.49 \mathrm{meV}$. (a), (b) Theory. (c), (d) Experiment. Simulation parameters: $\gamma_{X}=0.05 \mathrm{meV}, \beta / \alpha_{1}=0.5$. 

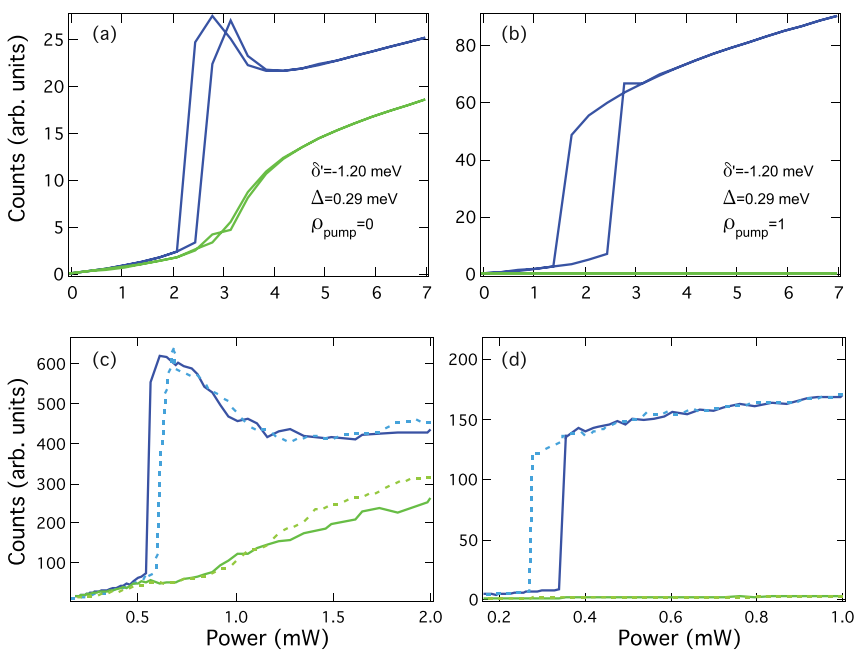

FIG. 14. (Color online) Comparison between experimental and theoretical bistability curves close to the exciton energy $\delta^{\prime}=$ $-1.2 \mathrm{meV}$. (a), (b) Theory. (c), (d) Experiment. Simulation parameters: $\gamma_{X}=0.3 \mathrm{meV}, \beta / \alpha_{1}=2$.

agreement. Even though there is some ambiguity in choosing the model parameters, the simulations indicate that it is not possible to reproduce the data at all detunings $\delta^{\prime}$ and $\Delta$ with a single value of the parameter $g_{R}$. We actually expect that the assumption that the blue shift depends linearly on the reservoir density is a poor approximation for high reservoir densities, because the blue shift is probably sublinear.

Because, to the best of our knowledge, the functional behavior of the blue shift on the reservoir density is not known, we keep using the linear dependence as a phenomenological model but adapt the value of $g_{R}$ when the detuning is changed (and thus the polariton density on the upper branch changes).

\section{DISCUSSION}

\section{A. The nature of the reservoir}

Our model only includes nonlinear losses of the form $\beta n_{\uparrow} n_{\downarrow}$. The experimental observation that led to this choice was the narrowing of the hysteresis width of the minority spin component when the majority density is high. We conjectured that the physical mechanism for this loss process is biexciton formation. The parameter $\beta$ that quantifies the nonlinear loss rate is seen to increase with increasing laser frequency, in agreement with our hypothesis that the biexciton resonance plays a prominent role in this physics.

The reproduction of the experimental results using our simulations shows that the blue shift $\Delta \epsilon=g_{R} n_{R}$ is of the order of the polariton line width. The feeding of the reservoir is determined by the nonlinear losses at a rate $P_{R}=\beta n_{\uparrow} n_{\downarrow}$. On the parameter $\beta$, there are restrictions from the measurements at a large polarization degree. The narrowing of the minority hysteresis [see, e.g., Fig. 10(b)] fixes quite precisely the ratio $\beta / \alpha_{1}$. This means that the creation rate of reservoir particles is fairly well known. Because the reservoir density is determined by the competition between creation and losses, the reservoir loss rate can be extracted fairly precisely as well. The resulting value, $\gamma_{R}=0.1 \mathrm{meV}$, is, however, much larger than the lifetime one would expect for biexcitons. It is actually expected to be an order of magnitude smaller, because only slow nonradiative losses contribute.

A longer lifetime of the biexcitons would require, in our model, a smaller value of the interaction constant $g_{R}$ by the same factor. The results of our theoretical model are roughly invariant under a replacement $g_{R} \rightarrow x g_{R}, \gamma_{R} \rightarrow \gamma_{R} / x$, where $x$ is a proportionality factor. With the current experimental data it is difficult to fix both parameters independently. A larger value of $\gamma$ is, however, more convenient numerically, because the dynamics reaches the steady state more rapidly. Physically, it is more likely that $\gamma_{R}$ is of the order of $0.01 \mathrm{meV}$ and that the effective $g_{R}$ is one order of magnitude smaller than the exciton-exciton interaction constant. This might be related to the decrease in $g_{R}$ with increasing reservoir density (see Sec. IV B).

\section{B. The value of $\alpha_{2}$}

An elementary question in the characterization of the spinor polariton system is the ratio of co- to counterpolarized interaction strengths $\alpha_{2} / \alpha_{1}$. Unfortunately, the present experiments do not give much information on this parameter. When two spin components are present, the reservoir builds up. This made it difficult to assess experimentally the interactions between two polaritons of opposite spin polarization in our experiments. For the fitting of the experimental data, we used $\alpha_{2} / \alpha_{1}=$ -0.1 , which gave satisfactory results. A zero interaction strength between two excitons of opposite polarization is indeed obtained within the Born approximation, where the true interaction strength is predicted to be negative. A negative value of $\alpha_{2}$ is confirmed by experiments in the parametric scattering regime. ${ }^{6}$ However, similar quantitative agreement could be obtained with a strictly zero value, $\alpha_{2}=0$.

\section{The independence of the lower thresholds}

For a single component optical field, the lower bistability threshold is reached approximately when the blue shift equals the laser detuning $\alpha_{1} n=\Delta$, where the density is then given by $n=I / \gamma^{2}$, so that the lower threshold power equals $I_{\mathrm{LT}}=$ $\Delta \gamma^{2} / \alpha_{1}$. In the case of a two-component nonlinear medium, two lower thresholds are present. Close to linear polarization, the difference in lower thresholds is mainly determined by the change in the nonlinear losses. Where the minority component (say $\sigma_{\downarrow}$ ) falls down, the line width is enhanced by the nonlinear losses and equals effectively $\gamma+\beta n_{\downarrow}$. On the other hand, where the majority component falls down, the line width is basically determined by the linear one, $\gamma$. The difference in lower thresholds can thus be estimated by

$$
\delta I_{\mathrm{LT}}=\frac{1}{\alpha_{1}}\left[\Delta_{\mathrm{eff}}^{(+)}\left(\gamma+\beta n_{\uparrow}^{(+)}\right)^{2}-\Delta_{\text {eff }}^{(-)} \gamma^{2}\right],
$$

where $n_{\uparrow}^{(+)}$is the density of polaritons in the majority component before the minority one falls down. Here $\Delta_{\text {eff }}^{(+/-)}$ denotes the effective laser detuning, when the system is on the upper/lower branch. It is reduced with respect to the bare laser detuning $\Delta$ due to the blue shift exerted by the reservoir on the polaritons.

The difference between the lower thresholds gives a strong experimental indication of biexciton resonance. In Fig. 15, 


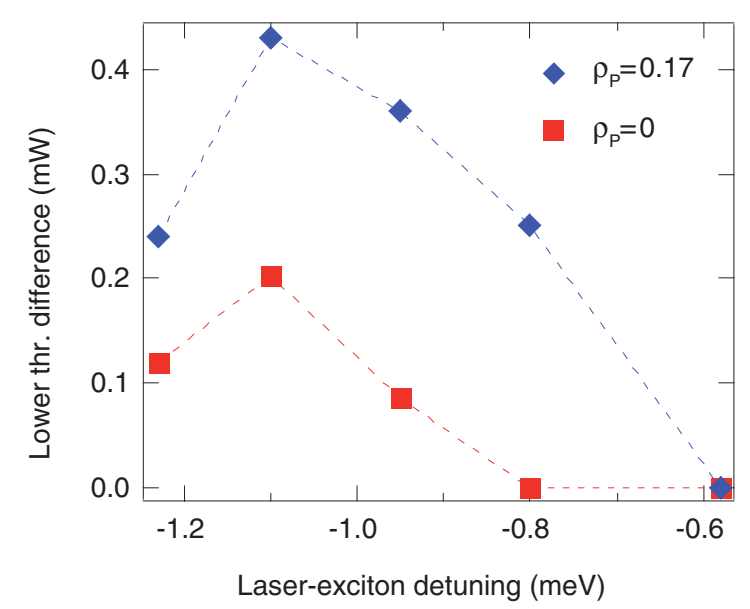

FIG. 15. (Color online) Polariton energy $\delta^{\prime}=-1.49 \mathrm{meV}$. The laser detuning with respect to the exciton $\Delta^{\prime}$ is changed from -1.24 to $-0.58 \mathrm{meV}$. For each value of $\Delta^{\prime}$, a series of spinor bistability experiments is performed. We record the lower threshold difference for linearly polarized excitation $\rho_{p}=0$ [(red) squares] and for elliptically polarized excitation $\rho_{p}=0.17$ [(blue) diamonds]. The threshold difference is enhancement when the laser energy is $1.1 \mathrm{meV}$ lower than the exciton energy, i.e., at the biexciton resonance.

we plot this difference as a function of the laser frequency at a fixed exciton-photon detuning. It shows a clear maximum at a laser-exciton detuning of $-1.1 \mathrm{meV}$, which is exactly where the biexciton resonance is expected. Indeed, the binding energy of the biexciton in our structure is around $2 \mathrm{meV}^{28}$ and a scattering resonance occurs when the total energy of the two scattering particles equals the energy of the bound state, i.e., for the single particle, at half the binding energy below the exciton energy. ${ }^{29}$ According to Eq. (7), this resonant behavior of $\delta I_{\mathrm{LT}}$ can be attributed to a resonance in the nonlinear loss rate $\beta$.

\section{The role of the polariton line width}

The power-dependent polarization switching behavior is sensitive to the polariton line width. For experiments where the polariton is close to the exciton and the line width is broad, no overlapping hysteresis loops are observed for the two polarizations. For a broader polariton line, a higher laser detuning is therefore needed to reach bistability, and consequently the polariton density on the upper branch is also higher. When the majority component jumps to the upper branch, the minority one sees its line width drastically increased so that it enters the optical limiter regime [see Fig. 14(a)]. Only at even higher laser detuning does the minority component start to show bistable behavior as well.

At large positive cavity detunings, the polariton line width becomes very large and polarization multistability can thus hardly be observed. Similar observations were made in our two-dimensional microcavity, as well as in the experiments by Sarkar et al. ${ }^{6}$ The narrow line width of mesas is actually one of their main advantages for achieving polarization multistability with respect to two-dimensional microcavities, where disorder and in-plane propagation cause broadening.

\section{POLARIZATION MULTISTABILITY}

Up to now, we have extensively discussed what can be learned from forward and backward laser power scans at fixed laser polarization. In the present section, we analyze in more detail the scans of the laser polarization at a fixed laser intensity. Such scans can give rise to polarization multistability: at zero laser polarization, three different polariton polarizations can exist for the system.

\section{A. Multistability in the $\left(n_{\uparrow}, n_{\downarrow}\right)$ plane}

A useful representation of the physics of multistability consists in reversing the nonlinear equations, calculating the parameters of the excitation field as a function of the polariton field. ${ }^{1}$ Figure 16 shows contours of fixed laser polarization in the polariton population plane $\left(n_{\uparrow}, n_{\downarrow}\right)$, computed with the SGPE. The light (dark) regions represent areas with positive (negative) laser polarization. In the two "anomalous" regions (labeled A1 and A2), the laser polarization is opposite to the polariton polarization. In the same graph, a single contour of fixed laser intensity is shown by the thick (red) line. The dynamically unstable regions of this contour are plotted by the dashed line. In Fig. 16 there are two separated curves, with the higher and lower polariton populations respectively. The closed curve corresponds to the system being on the low intensity branch of the power hysteresis cycle. During a polarization scan such as the one in Fig. 16(b), the system must follow the red line but simultaneously undergo a monotonic change of the laser polarization. When the thick (red) line enters the anomalous regions the system cannot follow continuously the red line without reversing the laser polarization variation. The red line becomes unstable and the system must jump to the closest stable region where the monotonic polarization change is possible [thin, dashed (green) lines in Fig. 16(a)]. In the stable regions of the thick (red) line, there are three points with zero laser polarization. Polarization multistability can then occur. The complete trajectory shows two jumps in (a)

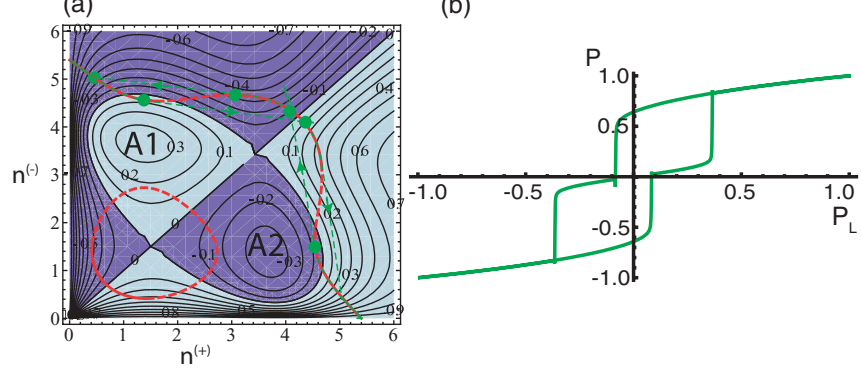

FIG. 16. (Color online) (a) Contour lines for constant laser polarization (thin lines) as a function of the polariton population in the two spin components $\left(n_{\uparrow}, n_{\downarrow}\right)$. Light (dark) shading indicates regions with positive (negative) laser polarization. The thick (red) line gives a single contour for a constant laser intensity. Dashed parts indicate the dynamically unstable regions of the contour. The thin, dashed (green) lines show the jumps in the trajectory of a scan of the laser polarization at a fixed laser intensity. (b) Polariton polarization along the laser polarization scan. Parameters for the SGPE: $\alpha_{2} / \alpha_{1}=0.1, \beta / \alpha_{1}=0.05, \Delta / \gamma=4, \alpha_{1} I_{L} / \gamma^{3}=16$. 
(a)

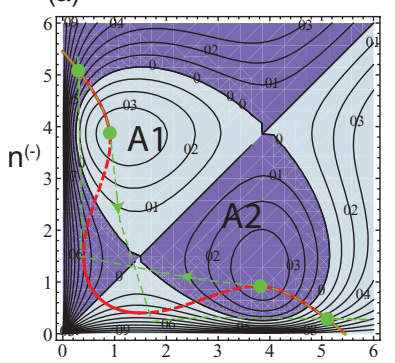

(b)

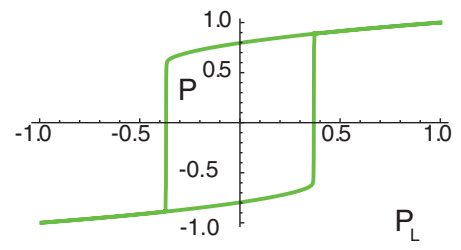

(c)

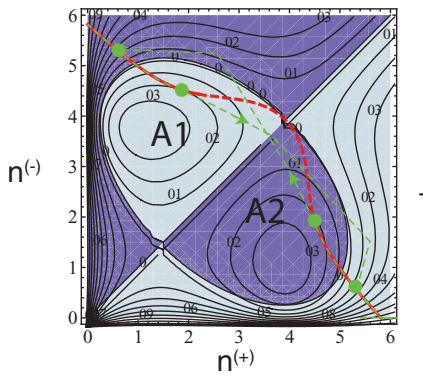

(d)

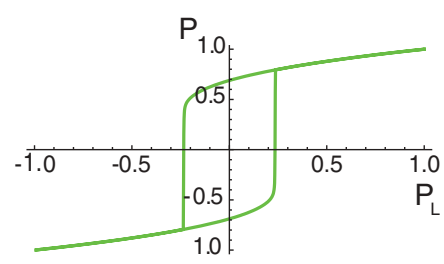

FIG. 17. (Color online) The same as Fig. 16, but for a larger value of $\beta / \alpha_{1}=0.3$. (a), (b) The laser intensity is the same as in Fig. 16; (c), (d) it is $\alpha_{1} I_{L} / \gamma^{3}=25$.

the backward and two jumps in the forward direction and a crossing between two of these jumps revealing multistability.

For increasing laser intensity, the red contour line moves to the upper right, out of the anomalous region (not shown). Polarization multistability therefore disappears at a high laser intensity. The effect of repulsive interactions between countercircularly polarized interactions is to decrease the size of the anomalous region, because the spin population which is favored by the laser is also helped by the other population. For the same reason, polariton-polariton attraction increases the domain of the multistability. Nonlinear polariton losses also increase the anomalous region, because the majority polaritons keep the minority density down through the nonlinear losses.

For larger nonlinear losses, the constant-intensity contour is qualitatively modified. The two separated curves can merge and form a single curve, as illustrated in Fig. 17. Following a polarization scan, the polariton polarization no longer stays close to zero polarization, but it jumps directly from high positive to high negative polarization. This is the so-called Schmidt trigger regime. Note that a zero polariton polarization region still exists, and experimentally, this "middle branch" can be reached by applying additional laser pulses.

When the laser power is increased for large nonlinear losses, the shape of the constant intensity contour changes [see Fig. 17(c)], but the evolution of the polariton polarization is qualitatively the same [see Fig. 17(d)].

\section{B. The role of nonlinear losses and the reservoir}

The phenomenon of polarization multistability depends crucially on the possibility for the polaritons to sustain, through hysteresis, a positive polarization when the laser polarization is already negative, i.e., the existence of anomalous regions A1 and A2. As discussed above, repulsive interactions tend to decrease the size of these regions, whereas attractive interactions and nonlinear losses tend to increase them.

The probably attractive polariton-polariton interactions are overcompensated by the effective repulsions through the buildup of a reservoir. This effect hinders the observation of multistability. Fortunately, the nonlinear losses that are associated with the creation of the reservoir increase the anomalous region, so that we can observe multistability in the region where the nonlinear losses are important, i.e., close to the biexciton resonance.

The other polariton energy where multistability could be expected is far away from any loss channel, so that the effective repulsive interaction through the reservoir is eliminated. In our sample, the Rabi frequency is, however, too low to have sufficient nonlinearity at detunings where the formation of the reservoir is expected to be negligible.

\section{CONCLUSIONS}

We have presented a detailed analysis of powerdependent polarization switching and polarization multistability in semiconductor microcavities. The comparison of the power-dependent switching experiments with the SGPE give a good qualitative agreement with the experimental observations when nonlinear losses are taken into account. We have argued that the most plausible mechanism for the nonlinear losses is through the formation of biexcitons.

Discrepancies between the experimental data and the predictions of the SGPE indicate the importance of the blue shift of the exciton due to the reservoir that is filled by the biexciton formation. Taking this blue shift into account gave a good quantitative agreement between theory and experiment and allowed us to fit the nonlinear loss parameter $\beta$, which indeed is seen to increase when the biexciton resonance is approached.

In our experiments on polarization multistability, the polariton polarization was followed on the Poincaré sphere. These experiments showed that the polaritons have very good coherence. The SGPE model reproduced the trajectory on the Poincaré sphere qualitiatively well. A further analysis of the conditions under which polarization multistability can occur provided strong evidence of a twofold role of the polariton reservoir. On the one hand, the exciton blue shift due to the reservoir blue shift counteracts multistability, but nonlinear losses favor multistability. This explains why, experimentally, multistability was only observed close to the biexciton resonance where the nonlinear losses are bigger.

\section{ACKNOWLEDGMENTS}

The authors thank N. A. Gippius, V. Kulakovskii, and G. Malpuech for fruitful discussions and O. El Daiff for his contributions in engineering the sample. The present work was supported by the Swiss National Research Fundation through "NCCR Quantum Photonics." M.W. acknowledges financial support from the ESF POLATOM network, FWO Odysseus G.0021.12, and LP.BOF.UA.2011. 
*Correspondence author: michiel.wouters@ua.ac.be

†taofiq.paraiso@a3.epfl.ch

${ }^{1}$ N. A. Gippius, I. A. Shelykh, D. D. Solnyshkov, S. S. Gavrilov, Y. G. Rubo, A. V. Kavokin, S. G. Tikhodeev, and G. Malpuech, Phys. Rev. Lett. 98, 236401 (2007).

${ }^{2}$ I. A. Shelykh, T. C. H. Liew, and A. V. Kavokin, Phys. Rev. Lett. 100, 116401 (2008).

${ }^{3}$ T. C. H. Liew, A. V. Kavokin, and I. A. Shelykh, Phys. Rev. Lett. 101, 016402 (2008).

${ }^{4}$ T. K. Paraïso, M. Wouters, Y. Léger, F. Morier-Genoud, and B. Deveaud-Plédran, Nat. Mater. 9, 655 (2010).

${ }^{5}$ C. Adrados, A. Amo, T. C. H. Liew, R. Hivet, R. Houdré, E. Giacobino, A. V. Kavokin, and A. Bramati, Phys. Rev. Lett. 105, 216403 (2010).

${ }^{6}$ D. Sarkar, S. S. Gavrilov, M. Sich, J. H. Quilter, R. A. Bradley, N. A. Gippius, K. Guda, V. D. Kulakovskii, M. S. Skolnick, and D. N. Krizhanovskii, Phys. Rev. Lett. 105, 216402 (2010).

${ }^{7}$ A. Amo, T. C. H. Liew, C. Adrados, R. Houdre, E. Giacobino, A. V. Kavokin, and A. Bramati, Nat. Photon. 4, 361 (2010).

${ }^{8}$ A. Amo, S. Pigeon, C. Adrados, R. Houdré, E. Giacobino, C. Ciuti, and A. Bramati, Phys. Rev. B 82, 081301 (2010).

${ }^{9}$ D. V. Vishnevsky, D. D. Solnyshkov, N. A. Gippius, and G. Malpuech, Phys. Rev. B 85, 155328 (2012).

${ }^{10}$ S. S. Gavrilov, A. S. Brichkin, A. A. Demenev, A. A. Dorodnyy, S. I. Novikov, V. D. Kulakovskii, S. G. Tikhodeev, and N. A. Gippius, Phys. Rev. B 85, 075319 (2012).

${ }^{11}$ S. Cecchi, G. Giusfredi, E. Petriella, and P. Salieri, Phys. Rev. Lett. 49, 1928 (1982).

${ }^{12}$ M. Kitano, T. Yabuzaki, and T. Ogawa, Phys. Rev. Lett. 46, 926 (1981).

${ }^{13}$ M. Haelterman, S. Trillo, and S. Wabnitz, J. Opt. Soc. Am. B 11, 446 (1994).
${ }^{14}$ F. Jonsson and C. Flytzanis, Phys. Rev. Lett. 82, 1426 (1999).

${ }^{15}$ K. Steiglitz, Phys. Rev. E 63, 046607 (2001).

${ }^{16}$ I. A. Shelykh, A. V. Kavokin, Y. G. Rubo, T. C. H. Liew, and G. Malpuech, Semicond. Sci. Technol. 25, 013001 (2010).

${ }^{17}$ P. G. Lagoudakis, P. G. Savvidis, J. J. Baumberg, D. M. Whittaker, P. R. Eastham, M. S. Skolnick, and J. S. Roberts, Phys. Rev. B 65, 161310 (2002).

${ }^{18}$ O. E. Daif, A. Baas, T. Guillet, J.-P. Brantut, R. I. Kaitouni, J. L. Staehli, F. Morier-Genoud, and B. Deveaud, Appl. Phys. Lett. 88 061105 (2006).

${ }^{19}$ R. Cerna, D. Sarchi, T. K. Paraïso, G. Nardin, Y. Léger, M. Richard, B. Pietka, O. El Daif, F. Morier-Genoud, V. Savona et al., Phys. Rev. B 80, 121309 (2009).

${ }^{20}$ A. Baas, J. P. Karr, H. Eleuch, and E. Giacobino, Phys. Rev. A 69, 023809 (2004).

${ }^{21}$ L. Cavigli and M. Gurioli, Phys. Rev. B 71, 035317 (2005).

${ }^{22}$ A. Tredicucci, Y. Chen, V. Pellegrini, M. Börger, and F. Bassani, Phys. Rev. A 54, 3493 (1996).

${ }^{23}$ D. Bajoni, E. Semenova, A. Lemaître, S. Bouchoule, E. Wertz, P. Senellart, S. Barbay, R. Kuszelewicz, and J. Bloch, Phys. Rev. Lett. 101, 266402 (2008).

${ }^{24}$ I. Carusotto and C. Ciuti, Phys. Rev. Lett. 93, 166401 (2004).

${ }^{25}$ M. Combescot, M. A. Dupertuis, and O. Betbeder-Matibet, Europhys. Lett. 79, 17001 (2007).

${ }^{26}$ V. Savona, J. Phys.: Condens. Matter 19, 295208 (2007).

${ }^{27}$ V. Savona, C. Piermarocchi, A. Quattropani, F. Tassone, and P. Schwendimann, Phys. Rev. Lett. 78, 4470 (1997).

${ }^{28}$ M. Saba, F. Quochi, C. Ciuti, U. Oesterle, J. L. Staehli, B. Deveaud, G. Bongiovanni, and A. Mura, Phys. Rev. Lett. 85, 385 (2000).

${ }^{29}$ N. H. Kwong, R. Takayama, I. Rumyantsev, M. Kuwata-Gonokami, and R. Binder, Phys. Rev. B 64, 045316 (2001). 